\title{
Communications Through WhatsApp by Medical Professionals
}

\author{
Varun Arunagiri $^{1}$ (D) $\cdot$ Kothai Anbalagan $^{2}$
}

Received: 30 June 2016/Accepted: 8 August 2016/Published online: 16 August 2016

(C) Association of Surgeons of India 2016

Sir,

I am glad to share the results of a questionnaire-based survey done using SurveyMonkey ${ }^{\mathrm{TM}}$ to assess the communications by medical professionals through WhatsApp. A questionnaire was formulated pertaining to the extent of use of WhatsApp by the medical professionals, and the e-link was circulated in the various social media like Facebook, Twitter, Viber, and emails for a period of 1 month by secured SSL encryption. All participants $(N=84)$ were medical professionals. The cohort had medical students $(21.43 \%)$, post diploma $(17.86 \%)$ and medical post graduates or residents $(27.38 \%)$, post graduates working in private sector $(13.1 \%)$, assistant professors in government medical colleges $(10.71 \%)$, and professors in private sector $(9.52 \%)$. Around $97.62 \%(n=82)$ agreed that they knew about WhatsApp. The entire cohort agreed to communicate through WhatsApp to their medical friends of the same cadre but not to their superiors $(11.9 \% ; n=10)$. Most of the medical professionals $(86.9 \%)$ had WhatsApp group for their batch. Medical professionals $(28.57 \% ; n=24)$ did not like to discuss about their patients or case discussion in WhatsApp. When the question was raised about the ethical issue that is it ethically acceptable to share about the patients' details in WhatsApp group, majority replied that they did not know $(42.86 \% ; n=36)$, the rest said that it is ethically unacceptable $(38.1 \% ; n=32)$, and few

Varun Arunagiri

surgeonvarun@yahoo.com

Madras Medical College, Institute of General Surgery, Chennai, TN, India

2 Chennai Corporation Hospital, Chennai, India
$(19.05 \% ; n=16)$ thought it is ethically acceptable. There was near equal priority to use WhatsApp in emergencies to call their colleague for help (yes vs no; 45.24 vs $54.76 \%$, respectively). A WhatsApp group by the medical professionals is preferred to discuss about the academics $(66.67 \% ; n=56)$ and journals $(23.81 \% ; n=20)$. Studies project the use of WhatsApp during emergencies [1], but among the medical professionals, it is a tool for discussion but not for treating the patient.

\section{Compliance with Ethical Standards}

Source of Funding None.

Conflict of Interest The authors declare that they have no conflict of interest.

\section{References}

1. Johnston MJ, King D, Arora S, et al. (2015) Smartphones let surgeons know WhatsApp: an analysis of communication in emergency surgical teams. Am J Surg 209:45-51 